\title{
Leiyomyosarcoma of the inferior vena cava with right renal invasion: about a case report
}

\author{
Abdoulazizi Bilgo ${ }^{1}$, Amine Saouli ${ }^{1 *}$, Ilyass Zerda ${ }^{1}$, Fouad Zouaidia ${ }^{2}$, Tarik Karmouni ${ }^{1}$, Khalid El Khader ${ }^{1}$, \\ Abdellatif Koutani ${ }^{1}$ and Ahmed Iben Attya Andalousi ${ }^{1}$
}

\begin{abstract}
Background: Leiomyosarcoma of the inferior vena cava (IVC) is a rare tumor that develops from the wall of the IVC and can be confused with many other retroperitoneal tumors. We report the observation of a man with leiomyosarcoma of the vena cava which invades the right kidney.

Case presentation: 56 -year-old man who has seen progress for right back pain for over a year. His thoraco-abdominal-pelvic scanner found a right tumor process measuring $18 \times 13 \times 18 \mathrm{~cm}$ invading the right kidney and the inferior vena cava, heterogeneous in nature, which is enhanced after injection of iodinated contrast product, pushing back the liver and the gall bladder. A border of separation persisted between the mass and the abdominal and thoracic walls. His biological assessment was normal. He underwent an open right nephrectomy with intraoperative bleeding requiring a transfusion of 2 red blood cells. The patient's follow-up period was 8 months without local recurrence or secondary localization.
\end{abstract}

Conclusion: The LMS of IVC is a tumor whose management is not yet well codified. Surgery is the only therapeutic means that gives good results, when it is possible. But long-term recurrences remain frequent, which therefore requires prolonged monitoring of these patients.

Keywords: Leiomyosarcoma, Inferior vena cava, Nephrectomy, Surgery

\section{Background}

Leiomyosarcoma of the inferior vena cava (IVC) is a rare tumor that develops from the wall of the IVC and can be confused with many other retroperitoneal tumors [1]. The diagnosis is often made by computed tomography (CT). A female predominance is noted in the literature. Surgery remains the only effective therapeutic means, but the tumor prognosis is poor in all cases. Chemotherapy has not yet demonstrated real efficacy on this tumor component [2]. Through this observation, we show the diagnostic and therapeutic peculiarities of this rare entity.

\footnotetext{
*Correspondence: amine.saoulio@gmail.com

${ }^{1}$ Department of Urology B, CHU Ibn Sina, Faculty of Medicine

and Pharmacy, Mohamed V University, Rabat, Morocco

Full list of author information is available at the end of the article
}

\section{Case presentation}

56-year-old man who has seen progress for right back pain for over a year. He had no family history and personally there is chronic smoking at a rate of 10 pack-years. This low back pain was associated with weight loss of around $5 \mathrm{~kg}$. Clinical examination found lumbar contact of the right flank without collateral venous circulation or superficial lymphadenopathy.

His thoraco-abdomino-pelvic scanner found: (Figs. 1, 2).

Abdomino-pelvic: an $18 \times 13 \times 18 \mathrm{~cm}$ right tumor process invading the right kidney and the inferior vena cava, heterogeneous in nature, which is enhanced after injection of iodinated contrast product, pushing back the liver and the gall bladder. A border of separation persisted between the mass and the abdominal and thoracic walls.

Thoracic stage: Presence of mediastino-hilar adenomegaly (5 lymphadenopathies) and diffuse centrilobular 


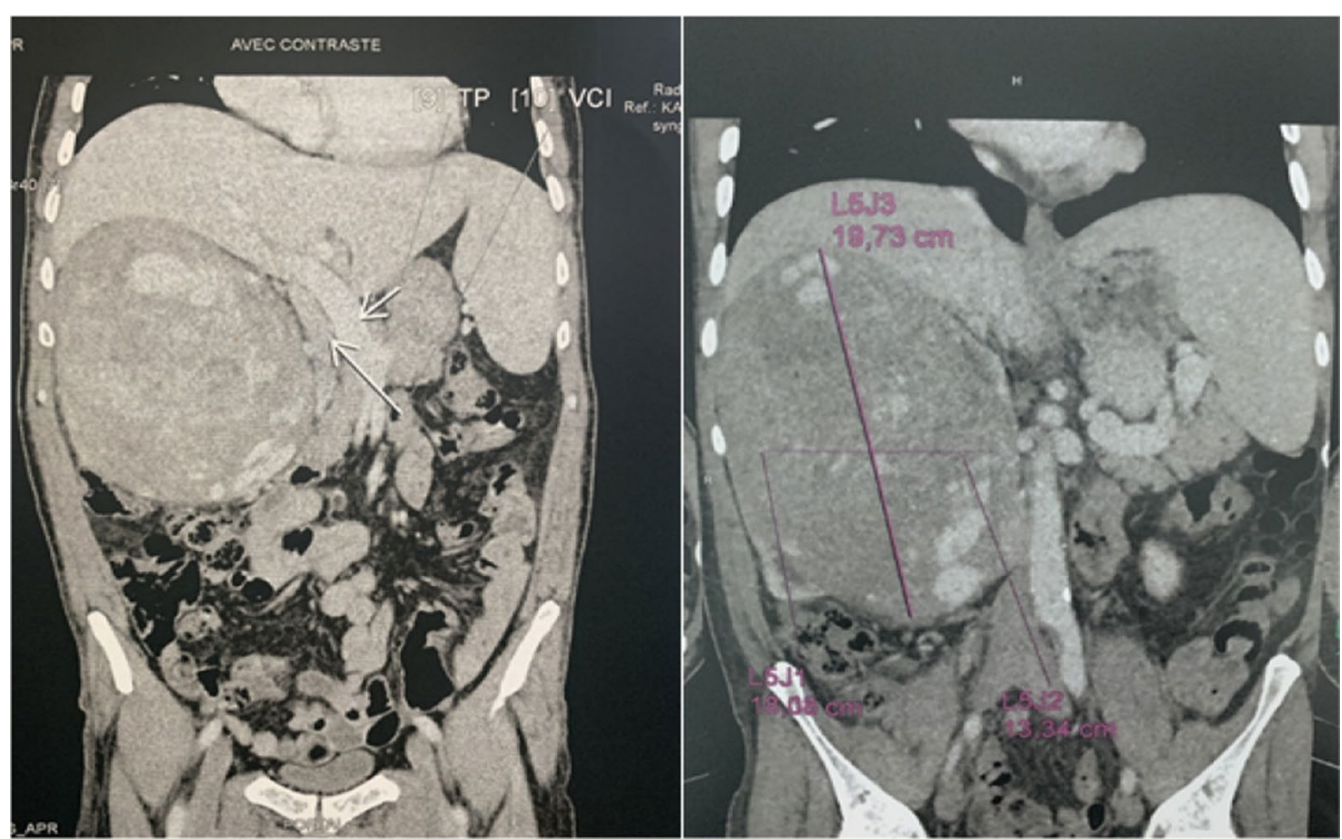

Fig. 1 Frontal section showing a large kidney tumor pushing back the IVC
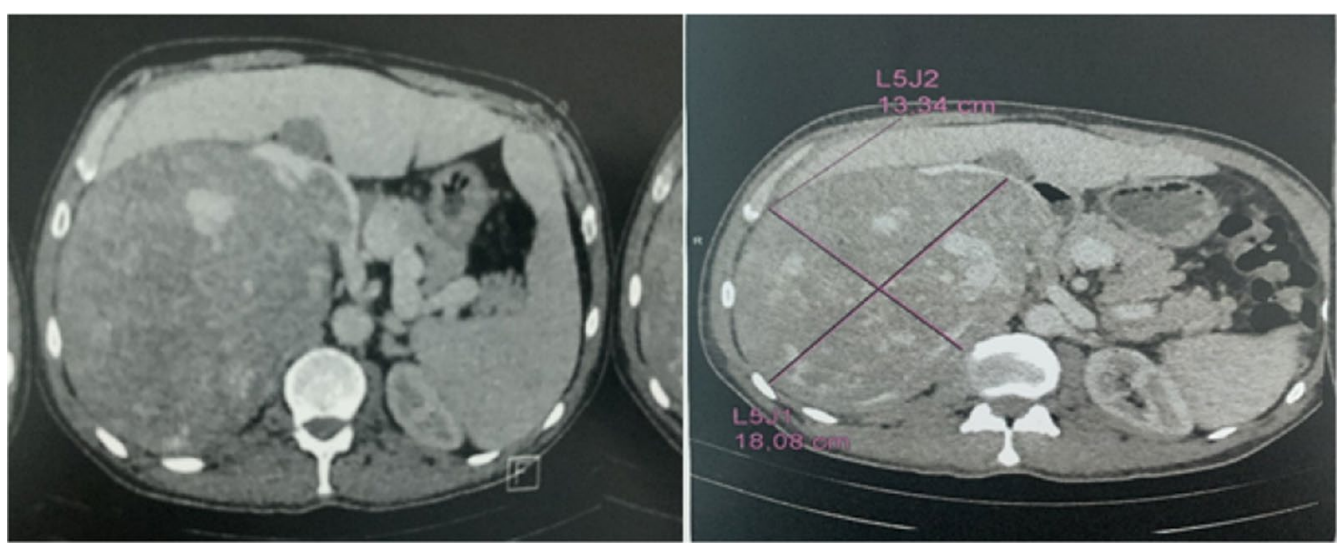

Fig. 2 Axial section showing a large renal tumor pushing back the IVC

micronodules, some of which presented with calcifications. The aspect remains equivocal between a secondary lesion and sequelae of tuberculosis.

His biological assessment found a hemoglobin $=12 \mathrm{~g} /$ $\mathrm{dl}$, a calcium level and normal LDH, a normal hepatic assessment and a creatinine clearance CKD-EPI $=75 \mathrm{ml} /$ $\min$.

We find an IMDC score $=2$ (haemoglobin < $13.5 \mathrm{~g} /$ $\mathrm{dl}$ and time between diagnosis and systemic treatment < 1 year), so this is an intermediate risk.

He underwent a right subcostal nephrectomy with resection of an invaded IVC flap. An intraoperative hemorrhagic incident led to the transfusion of 2 red blood cells.

The pathological result was in favor of a leiomyosarcoma of the inferior vena cava (Fig. 3) invading the right renal vein as well as the kidney. The microscopic appearance was characterized by the presence of spindle-shaped cells in the eosinophilic cytoplasm with elongated nuclei with clumped chromatin. The surgical margins of the part and the recut of the IVC passed into the healthy zone. 

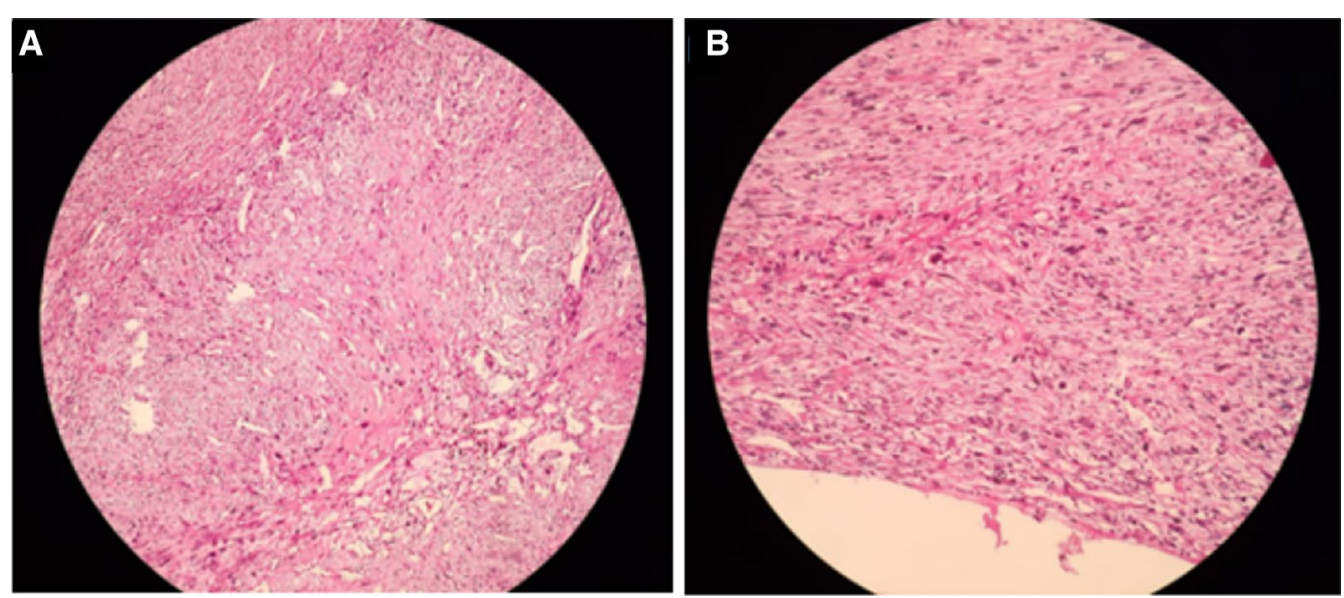

Fig. 3 Microscopic section of leiomyosarcoma of IVC with a G 10 HE (a) and G 20 HE (b)

The immunohistochemistry found anti-AML, anti$\mathrm{H}$ caldesmone, anti-desmine positive and anti-CD34 negative.

The patient's follow-up period was 8 months without local recurrence or secondary localization.

\section{Discussion}

LMS is a malignant tumor that grows at the expense of smooth muscle fibers in the vascular wall. It accounts for $6 \%$ of all sarcomas. It involves the inferior vena cava (IVC) in $38 \%$ of cases and constitutes $95 \%$ of primary tumors in IVC [3]. In 1871, Perl et al. the very first description of this location is post mortem. Since then, a few small series have been described but above all case reports. An international IVC sarcoma registry opened in 1992 has identified 218 cases and 418 cases reported globally in the literature [4].

This is a clearly predominantly female pathology with a sex ratio of $1 / 5$, and which often occurs from the 5 th decade onwards. It has a slow evolution, and when it is symptomatic, it is revealed by low back pain in more than $80 \%$ of cases [2]. In this case, the main symptom was low back pain.

The proliferative power of the LMS of the IVC is low with a little infiltration character. Its metastatic dissemination is slow and takes place systemically to the liver and lungs; however, lymphatic dissemination remains quite rare [5]. Our patient CT-scan didn't fine any lymphadenopathy in abdominal stage.

CT with injection of iodinated contrast medium (ICM) is the gold standard examination to show a retroperitoneal mass encapsulating the inferior vena cava. However, the diagnosis between LMS of IVC and any other retroperitoneal sarcoma sometimes remains difficult on imaging, especially if its extraluminal component is predominant.

A few CT signs guide the diagnosis [6]:

- A tumor that invades and dilates the lumen of the IVC is almost pathognomonic of an LMS of the IVC.

- The sign of being embedded in an organ "Positive embedded IVC sign": the IVC is stuck in the mass and not repressed.

- The sign of the imperceptible IVC "Imperceptible cava lumen": the IVC is not visualized at the point of maximum contact with the tumor mass. This sign is most useful according to Webb et al. in identifying a retroperitoneal mass such as leiomyosarcoma from IVC.

The tumor mass is most often lateralized on the right. This right lateralization during its growth is explained by the lower resistance of the fatty tissue on the right compared to the aorta on the left [2]. The imagery data allowed Kulaylat et al. to establish in 1997 a classification of LMS according to their location the different segments of the IVC [7]: (Fig. 4).

- Segment I (infra-renal) corresponding to the IVC below the entrance to the renal veins.

- Segment II (middle segment) corresponding to the IVC located between the end of the hepatic veins and the renal veins.

- Segment III (hepatic) corresponding to the IVC located between the right atrium and the hepatic veins.

The most frequent localization concerns segment II. The patient's symptoms therefore relate to the affected 


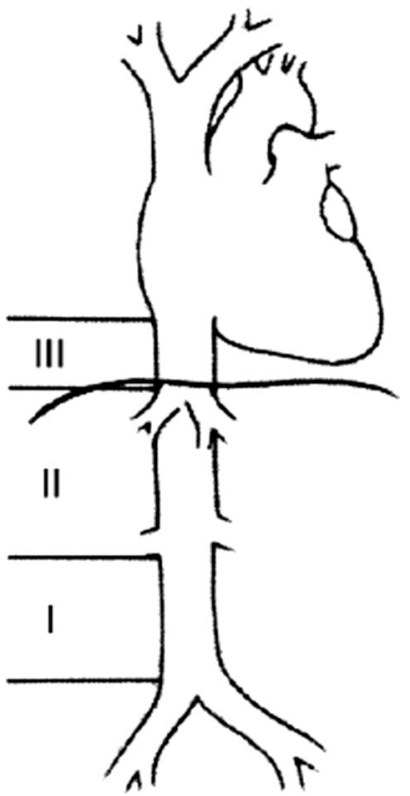

Fig. 4 A classification of LMS according to their location the different segments of the IVC

segment, but also to the tumor volume and its growth kinetics [7, 8]. In this case, it was difficult to clearly assess the origin of the tumor, either the IVC or kidney. Our diagnosis was made on the basis of the partial invasion of the IVC and the absence of local lymphadenopathy contrasting with a large mass. Although the renal vein may be the origin of the tumor, but with a very low incidence, the imaging of our patient remains ambiguous, also given the invasion of the inferior vena cava.

The role of MRI remains limited to differentiating a neoplastic invasion from a thrombus in case of doubt, or when CT with injection of ICM is contraindicated [5].

A biopsy is not required if the mass is removable. It remains an indication in non-operable patients for possible systemic therapy or in case of doubtful diagnosis (lymphoma, adrenal tumor, history of neoplasia in the patient).

The only treatment that has proven to be effective is monobloc excision of the tumor in healthy margins if necessary with enlargement to neighboring organs [5]. LMS excision for IVC was first performed in 1951 at the Lexington Memorial Hospital in Chicago [8]. The principle of this management consists in performing an R0 excision of the tumor, ensuring venous return of the territories concerned and preventing recurrence $[3,5]$. Our patient underwent radical nephrectomy with resection of an invaded IVC flap.

The difficulty of tumor resection depends on the location of the LMS. Tumors located in segment I of the IVC are accessible to complete resection without difficulty because complete clamping of the infra-renal IVC is often well tolerated. The excision of segment III tumors is conditioned by the invasion of the hepatic veins and is often impossible to perform. In segment II, surgery has to deal with two problems: the retrohepatic location of the tumor and the possible invasion of one or both renal veins [3]. The complexity of the surgical procedure sometimes requires the availability of a multidisciplinary team and an expert center.

The role of chemotherapy and radiotherapy (neoadjuvant or adjuvant) remains to be specified. For a resectable tumor, no data in the literature demonstrate the benefit of neoadjuvant chemotherapy [2].

Histology reveals a fasciculated proliferation of elongated, spindle-shaped cells arranged in a brush, the malignant character of which is retained on the appearance of the nuclei. Immunohistochemistry was found to be positive for smooth muscle actin, desmin and CD34. CD117 labeling is negative and the Ki-67 proliferative index remains low [4]. About our patient, the diagnosis of leiyomyosarcoma was made on the basis of immunohistochemistry. The entire renal vein and the inferior vena cava flap were invaded. Although we have mentioned an origin from the IVC, an origin from the renal vein could not be ruled out.

The evolution of LMS in IVC remains pejorative with a 5-year survival rate of less than $50 \%$ and a 10 -year survival rate of less than $30 \%$. The major problem with these tumors is recurrence, which occurs in one in two to one in three cases with a median at 25 months $[3,5,8]$.

Factors that influence short-term survival are [2]:

- A level III tumor.

- The extension to the right atrium.

- The presence of an endoluminal tumor bud.

- Macroscopically incomplete surgery.

\section{Conclusion}

LMS is a rare tumor but even rarer in men. Sometimes the diagnosis remains difficult as well with the CT-scan as with the histology as was the case of our patient. Its management is not yet well codified. Surgery is the only therapeutic means that gives good results, when it is possible. But long-term recurrences remain frequent, which therefore requires prolonged monitoring of these patients.

\section{Abbreviations}

LMS: Leiomyosarcoma; IVC: Inferior vena cava; CT: Computed tomography; ICM: lodinated contrast medium. 
Acknowledgements

Not applicable.

\section{Authors' contributions}

$A S, A B$ and $I Z$ analyzed and interpreted the patient data regarding the subject. $F Z, T K, K E, A K$ and AIAA were a major contributor in writing the manuscript. All authors read and approved the final manuscript.

\section{Funding}

No funding.

\section{Availability of data and material}

Not applicable.

\section{Declarations}

\section{Ethics approval and consent to participate}

The ethics committee of the Faculty of Medicine of Rabat has given us its agreement. Informed consent to participate in the study was provided by the patient. The reference number is not applicable.

\section{Consent for publication}

The patient gave his informed and written consent for the publication of this work.

\section{Competing interests}

The authors declare that they have no conflicts of interest in connection with this article.

\section{Author details}

'Department of Urology B, CHU Ibn Sina, Faculty of Medicine and Pharmacy, Mohamed V University, Rabat, Morocco. ${ }^{2}$ Department of Pathological Anatomy, CHU Ibn Sina, Faculty of Medicine and Pharmacy, Mohamed V University, Rabat, Morocco.
Received: 17 March 2021 Accepted: 12 June 2021

Published online: 24 June 2021

\section{References}

1. Sephien A et al (2019) Leiomyosarcoma of the inferior vena cava with hepatic and pulmonary metastases: case report. J Radiol Case Rep 13(5):30-40

2. Sulpice $L$ et al (2016) Leiomyosarcoma of the inferior vena cava. J Visc Surg 153(3):161-165

3. Bonnet $\mathrm{S}$ et al (2006) Leiomyosarcoma of the middle segment of the infeerior vena cava: tactical problems raised by renal and caval revascularisation. J Mal Vasc 31(2):79-84

4. Sessa B et al (2010) Imaging of leiomyosarcoma of the inferior vena cava: comparison of 2 cases and review of the literature. Cancer Imaging 10:80-84

5. Hammoune $\mathrm{N}$ et al (2015) Leiomyosarcoma of the inferior vena cava: a case report. Pan Afr Med J 20:283

6. Webb EM, Webb Z, Westphalen AC, Nakakura EK, Yeh BM, Coakley FV (2013) Can CT features differentiate between inferior vena cava leiomyosarcomas and primary retroperitoneal masses? AJR Am J Roentgenol 200(1):205-209

7. Kulaylat MN et al (1997) Leiomyosarcoma of the inferior vena cava: a clinicopathologic review and report of three cases. J Surg Oncol 65(3):205-217

8. Teixeira FJR Jr et al (2017) Leiomyosarcoma of the inferior vena cava: survival rate following radical resection. Oncol Lett 14(4):3909-3916

\section{Publisher's Note}

Springer Nature remains neutral with regard to jurisdictional claims in published maps and institutional affiliations.

\section{Submit your manuscript to a SpringerOpen ${ }^{\circ}$ journal and benefit from:}

- Convenient online submission

- Rigorous peer review

- Open access: articles freely available online

- High visibility within the field

- Retaining the copyright to your article

Submit your next manuscript at $\boldsymbol{\nabla}$ springeropen.com 